\title{
Adoption of management decisions on the basis of the risk management model
}

\author{
Galina Skachko ${ }^{1, *}$, Ekaterina Surkova ${ }^{1}$,Elena Ermolaeva ${ }^{1}$, and Irina Pocebneva ${ }^{2}$ \\ ${ }^{1}$ Moscow Aviation Institute (National Technical University), Volokolamckoe shosse, 4, Moscow, \\ 125993, Russia \\ ${ }^{2}$ Voronezh State Technical University, 20 years of October st., 84, Voronezh, 394006, Russia
}

\begin{abstract}
The construction of a dynamic model of risk management for a construction company is considered, which can significantly increase the efficiency of management decisions. To build a dynamic model, the most common approaches to management are considered: process, system and situational. To effectively assess the risks of a construction company, a two-stage model for assessing static and dynamic indicators was used. At the initial stage, the probability of risk values for groups of factors (financial, engineering, technological, economic, marketing) was determined. At the second stage, the degree of influence of groups of factors on the overall probability of risk occurrence is determined. As a result, for each of the analyzed indicators that determine the risk, a sequence of its most probable values is formed at the moment $\mathrm{t}_{\mathrm{k}}$.
\end{abstract}

\section{Introduction}

The management of complex organizational systems to which construction companies can be attributed is based on the application of principles and methods for achieving goals that presuppose the co-operation of executive activity based on the realization of managerial functions. To achieve the goals of management, it is also necessary to develop general patterns of implementation of management activities that contribute to the formation of sustainable decision-making schemes.

The development of a management system in accordance with the requirements of the international standard ISO 9001: 2015 series focused on the production of quality products that meets the requirements of consumers is the key to the competitiveness of the enterprise.

There are three most common management approaches: process, system and situational.

In the process approach, management functions are considered not as autonomous, but as organically interrelated and forming in their totality a single management process. At the same time, the management process is considered as a chronologically ordered and cyclically organized system of management functions. Management functions can be grouped into four main categories: planning, organization. motivation and control.

\footnotetext{
* Corresponding author: skachko_galina@mail.ru
} 
Consequently, the main condition for successful management is not only the efficiency of managerial functions, but also their correct co-organization in a single process.

The system approach assumes the perception of an organization as a single integral structure consisting of interconnected parts that include qualitatively heterogeneous components (equipment, technology and people). At the same time, it is necessary to take into account that any, even private, managerial influence on any component of the system directly or indirectly affects the achievement of management objectives. Any organization as a system has its own internal laws that must be considered when making management decisions. The system approach forms a new understanding of the organization as a sociotechnical system, the management of which should be based on making management decisions based on a comprehensive assessment of the dependency of the system components.

The situational approach also develops one of the main theses of the system approach, supplementing it with the statement that any organization is an open system that is in constant interaction with the external environment (informational, energy, material). Consequently, the main reasons for internal laws should be sought in the external situation in which it actually functions. Under the situation understand the specific system of circumstances and conditions that most strongly affect the organization at a given time.

For the most successful achievement of the objectives of the organization in a variety of life situations, the manager needs a synthesis of heterogeneous knowledge, as well as the ability to choose the best methods and methods of management.

In accordance with the situational approach, the management process can be divided into four stages:

1. the formation of the managerial competence of the head, i.e. the degree of his ownership of controls;

2. anticipation of possible consequences (both positive and negative);

3. adequate interpretation of the situation (identification of its main factors, the so-called situational variables, both external and internal, as well as an assessment of the effectiveness of the impact on one or several variables);

4. coordination of management techniques selected by a manager with specific conditions based on the requirement of maximizing positive and minimizing negative effects.

The most significant stage can be called the stage of choosing the most significant factors for the situation, both external and internal variables that can affect the system. With the wrong choice of situational variables or the wrong impact on them, there is a risk of an adverse event that can affect the system's ability to achieve its intended goals.

\section{Experimental}

Risk management is a risk-based activity aimed at reducing the likelihood of a risk event occurring to a minimum level, taking into account the constraints imposed on resources and time. For an effective risk assessment of a building enterprise, we use a two-stage assessment model with regard to static and dynamic indicators.

At the initial stage, we will determine the probabilities of risk values for groups of factors (financial, engineering, technological, economic, marketing) [1];

at the second stage, we will determine the degree of influence of groups of factors on the overall probability of risk occurrence.

Calculation of the probability of risk values for groups of factors. The risk level of a construction enterprise is determined by $\underset{m \in M}{\forall} X_{m}=\left\{X_{k}^{m}: k \in 1, K_{m}\right\} \neq 0$, determine each and collectively the overall integral assessment of the quality of work performed. Let there be a 
set of data on specific estimates of risk parameters for a time period $t_{0} ; t^{*} ; t_{k}$, where $t_{0^{-}}$ forecast period; $t^{*}$ - starting point; $t_{k^{-}}$forecast period.

It is necessary to take into account that the parameters themselves that determine the level of risk are not random values and are determined on the basis of financial and regulatory documents of the construction enterprise both in groups and in results. In this connection, in this task we can only determine the probability of the risk value [2] at the moment of time $\boldsymbol{t}_{\boldsymbol{\kappa}}$.

As the initial data we take the values of the parameters that cause the risk of the construction company $-\boldsymbol{X}_{\boldsymbol{I}}{ }^{\boldsymbol{T}}$.

Then we determine the number of different outcomes. $\boldsymbol{a}_{\boldsymbol{j} i}$, groups of values can be divided according to the degree of achievement of the desired result. Then, $\boldsymbol{a}_{1 \boldsymbol{i}}-$ number of high risk outcomes $\boldsymbol{X}_{1}{ }^{1} ; \boldsymbol{a}_{2 i}$ - number of risky outcomes $\boldsymbol{X}_{\boldsymbol{1}}{ }^{\boldsymbol{l}} ; \boldsymbol{a}_{3 i}$ - number of medium risk situations $\boldsymbol{X}_{\boldsymbol{I}} \boldsymbol{I} ; \boldsymbol{a}_{3 \boldsymbol{i}}$ - number of low risk outcomes $\boldsymbol{X}_{\boldsymbol{I}}{ }^{\boldsymbol{}} ; \boldsymbol{a}_{\boldsymbol{m} \boldsymbol{i}}$ - the number of outcomes with no risk $\boldsymbol{X}_{\boldsymbol{I}}$. Then the frequency of obtaining the results of the samples according to the degree of achievement will be distributed as follows:

$$
\begin{aligned}
& p_{1}^{*}=\frac{a_{1 i}}{m} ; p_{2}^{*}=\frac{a_{2 i}}{m} ; p_{3}^{*}=\frac{a_{3 i}}{m} ; \\
& p_{4}^{*}=\frac{a_{4 i}}{m} ; p_{m}^{*}=\frac{a_{m i}}{m}
\end{aligned}
$$

where $p_{1}^{*} ; p_{2}^{*} ; \ldots p_{m}^{*}$ - frequencies of high risk, risky, medium risk, low risk and no risk outcomes for financial indicators $\boldsymbol{X}_{\boldsymbol{I}} \boldsymbol{I}$ during the period $\left[t_{0} ; t^{*}\right]$.

Suppose the random variable $\boldsymbol{X}_{\boldsymbol{I}}{ }^{1}$ distributed according to the normal law [3] with $\boldsymbol{m}-\boldsymbol{I}$ degree of freedom. Then, as a point estimate for the unknown probability of events $p_{1} ; p_{2} ; \ldots p_{m}$ use frequency wisely $p_{1}^{*} ; p_{2}^{*} ; \ldots p_{m}^{*}$. However, then it is necessary to set the accuracy and reliability of such an assessment.

For large values $\boldsymbol{m}$ event frequency $\boldsymbol{a}_{j i}$ seeks to normal law. In this case, the condition: $n p>4$ and $n q>4$, where $q=1-p$ - the likelihood that an event will not occur. Since these conditions are met in our case, and the frequency $\boldsymbol{p}^{*}$ distributed according to the normal law, then the following parameters apply:

- expected value for a group of indicators $X_{I}{ }^{1}$ :

$$
m_{x_{1}^{1}}=\frac{1}{m} \sum_{i=1}^{m} x_{i j} p_{i}^{*}
$$

where $\boldsymbol{x}_{i j}$ - parameter value of a random variable $\boldsymbol{X}_{\boldsymbol{I}}{ }^{1}$;

- standard deviation for $\boldsymbol{X}_{\boldsymbol{I}}{ }^{1}$ :

$$
\sigma_{p^{*}}=\frac{1}{m-1} \sqrt{\left(x_{i j}-m_{x}\right)^{2}}
$$

In this case, the condition:

$$
\begin{aligned}
& m_{p}{ }^{*}=p \\
& \sigma_{p^{*}}=\sqrt{\frac{p q}{n}}
\end{aligned}
$$


To determine the probability of outcomes, the confidence probability is $\beta$, which shows the degree of possibility of hitting the confidence interval $\left|p^{*}-p\right|<\xi_{\beta}$ to the desired probability [4]. For the task of forecasting the level of risk of the construction company for the period $\left[t^{*} ; t_{k}\right]$ the confidence probability is set equal to 0,8 .

Thus, the task of predicting the level of risk is reduced to finding the probability of a given event occurring at a known random value $\boldsymbol{x}_{i j}(0,2 ; 0,3 ; 0,5 ; 0,7 ; 1)$.

Since the value $p^{*}$ distributed normally then:

$$
P\left(\left|p^{*}-p\right|<\xi_{\beta}\right)=\beta
$$

(a $\boldsymbol{\beta}=0,8)$ a $\xi_{\beta}=\sigma_{p} * \operatorname{arq} \Phi^{*}\left(\frac{1+\beta}{2}\right)$, then denoting $t_{\beta}=\operatorname{arq} \Phi+\left(\frac{1+\beta}{2}\right)$ (the number of standard deviations that need to be delayed to the right and to the left of the center of dispersion in order that the probability of getting into the resulting region is equal to the given).

We determine the value of the confidence probability through the following relation:

$$
\xi_{\beta}=t_{\beta} \sigma_{p^{*}}
$$

Knowing the expression for determining the standard deviation, and the value of the tabulation function is taken from the table [4], it is possible to equate both sides for a confidence level. Then:

$$
\text { 1. } \left.\left|p^{*}-p\right| \approx t_{\beta} \sigma_{p}=t_{\beta}\left(\frac{1}{m-1} \sqrt{\left(x_{i j}-m_{x}\right.}\right)^{2}\right) .
$$

As a result of the transformations we get:

$$
\left.p=p^{*}-t_{\beta}\left(\frac{1}{m-1} \sqrt{\left(x_{i j}-m_{x}\right.}\right)^{2}\right)
$$

Analyzing the dependence obtained, one can come to the following conclusions:

- the probability of occurrence of the desired event in the forecast period cannot exceed the value of the confidence probability;

- with a significant increase in the number of observations $m \rightarrow \infty$ the probability values of the event and the confidence probabilities coincide;

- an increase in the value of the confidence probability above 0.8 is undesirable, since the confidence interval increases and thus the accuracy of the forecast is reduced.

Now we calculate the values of the probability of occurrence of risk at the moment $\boldsymbol{t}_{\boldsymbol{k}}$ for options (high risk, high risk, medium risk, low risk and no risk outcomes) [6]:

- high risk forecast:

$$
\begin{aligned}
& p_{1}=p_{1}{ }^{*}-t_{\beta} \\
& \left.\left(\frac{1}{m-1} \sqrt{\left(0,2-\frac{\left(0,2 a_{1 j}+0,3 a_{2 j}+0,5 a_{3 j}+0,7 a_{7 j}+1 a_{m j}\right)}{m}\right.}\right)^{2}\right)
\end{aligned}
$$

- risk forecast: 


$$
\begin{aligned}
& p_{2}=p_{2}{ }^{*}-t_{\beta} \\
& \left(\frac{1}{m-1} \sqrt{\left.\left(0,3-\frac{\left(0,2 a_{1 j}+0,3 a_{2 j}+0,5 a_{3 j}+0,7 a_{7 j}+1 a_{m j}\right)}{m}\right)^{2}\right)}\right.
\end{aligned}
$$

- medium risk forecast:

$$
\begin{aligned}
& p_{3}=p_{3}{ }^{*}-t_{\beta} \\
& \left(\frac{1}{m-1} \sqrt{\left.\left(0,5-\frac{\left(0,2 a_{1 j}+0,3 a_{2 j}+0,5 a_{3 j}+0,7 a_{7 j}+1 a_{m j}\right)}{m}\right)^{2}\right)}\right.
\end{aligned}
$$

- low risk forecast:

$$
\begin{aligned}
& p_{4}=p_{4}{ }^{*}-t_{\beta} \\
& \left(\frac{1}{m-1} \sqrt{\left.\left(0,7-\frac{\left(0,2 a_{1 j}+0,3 a_{2 j}+0,5 a_{3 j}+0,7 a_{7 j}+1 a_{m j}\right)}{m}\right)^{2}\right)}\right.
\end{aligned}
$$

- reference forecast:

$$
\begin{aligned}
& p_{5}=p_{5}{ }^{*}-t_{\beta} \\
& \left(\frac{1}{m-1} \sqrt{\left(1-\frac{\left(0,2 a_{1 j}+0,3 a_{2 j}+0,5 a_{3 j}+0,7 a_{7 j}+1 a_{m j}\right)}{m}\right)^{2}}\right)
\end{aligned}
$$

Then, according to the presented algorithm, the forecast is calculated for other indicators [7]. For each of the calculated parameters, the forecast value that has the highest probability is selected. As a result, for each of the analyzed indicators that determine the risk, a sequence of its most probable values is formed at the moment of $\boldsymbol{t}_{\boldsymbol{k}}$.

Calculation of the degree of influence of individual factors and their groups on the overall probability of occurrence of risk [7].

Now it is necessary to establish the strength of the influence of individual factors, the degree of influence of individual factors and their groups on the overall probability of occurrence of risk.

The object of study will be the risk function.

The risk of a building enterprise determines many factors.:

$$
\forall_{m \in M} X_{m}=\left\{X_{k}^{m}: k \in 1, K_{m}\right\} \neq \varnothing
$$

Let the set of all factors implemented in the system be defined: $X^{m}=\left\{X_{m}: m \in\{1, M\}\right.$, где $M \geq 1$ means the number of risk factors, and for each risk, many of its elements are defined [8]: $M^{h}=\left\{m: \wedge P^{m} \in P_{h}\right\}, \bigcup_{k=1} M^{h}=M$ Then we get a family of sets: $M^{H}=\left\{M^{h}: h\right.$ $\in\{1, M\}_{\}}$.

The dynamic structure is set: $\left(P^{H}, R_{H}\right), R_{H} \subset P^{H} \cdot P^{H}$, where is the number $H$ determines the degree of its complexity. If $N=1$, then the dynamic structure is minimal if $N=M$, she is maximal. Moreover, if the elements of the set $M_{f}, f \in 1, F$, implement $f$-ю the function of the system, they implement the elementary factors of the same kind. 
Wherein $F$ - the number of different factors implemented in the risk management system [9]. Then structure $S_{f}=\left\{M^{F}, R_{F}\right), R_{F} \subset M^{F} \cdot M^{F}$ will be functional. If $F=1$, This functional structure is minimal or, in other words, the maximum concentration of the function in the risk management system. If a $F=M$, then the maximum deconcentration (specialization) of the function in the risk management system [10].

To ensure a favorable forecast of goals, the following condition must be met:

$$
\underset{t_{0} \in T}{\wedge} \underset{t_{k} \in T}{\vee} S_{M}\left(t_{0}\right) \neq S_{M}\left(t_{k}\right) \vee S_{F}\left(t_{0}\right) \neq S_{F}\left(t_{k}\right)
$$

Functionally, the ability to achieve the goals of a risk management system will highlight extreme combinations of options when changes over time cause functional integration in the risk management system (goals achieved) or functional disintegration (goals are not achieved) [11]. The process of achieving goals is real if for $t_{0} \rightarrow t_{k}, t_{0}<t_{k}, S_{F_{0}}\left(t_{0}\right) \rightarrow S_{1}\left(t_{k}\right)$ т т $F_{0} \rightarrow 1$ for $1<F_{0} \leq M$; and the process of achieving goals is unreal if for $t_{0} \rightarrow t_{k}, t_{0}<t_{k}, S_{F_{0}}\left(t_{0}\right) \rightarrow S_{M}\left(t_{k}\right)$ т т $F_{0} \rightarrow M$ for $1<F_{0} \leq M$.

\section{Evaluation}

Adaptive structure of the risk management system is the dependence of the type [12]:

$$
S_{R} \equiv\left(X^{*} ;\left(S\left(t_{0}\right)\right), S\left(t_{k}\right), \Delta\left(t_{0}, t_{k}\right)\right)
$$

(where: $X$ - multiple inputs (goals achievement factors; $T$ - forecast period.

$$
\begin{aligned}
& t_{0}<t_{k}, t_{0}, t_{k} \in T \\
& S\left(t_{0}\right) \equiv\left(S_{M}\left(t_{0}\right), S_{F}\left(t_{0}\right)\right) ; S\left(t_{k}\right) \equiv\left(S_{M}\left(t_{k}\right), S_{F}\left(t_{k}\right)\right) ;
\end{aligned}
$$

$\Delta\left(t_{0}, t_{k}\right)=v\left(t_{k}\right)-v\left(t_{0}\right) ; v\left(t_{0}\right), v\left(t_{k}\right) \in V$ где $v\left(t_{0}\right), v\left(t_{k}\right)$ - effects of reaching goals at times $t_{0}, t_{k}$ ) [13-14]. Also, an adaptive risk management system can be described by the following qualitative characteristics: if $X^{*} \subset \bar{Y}$, then adaptation takes place under the influence of the environment; if a $X^{*} \subset Y$, it is an independent adaptation; if a $X^{*} \subset Y \times \bar{Y}$, then adaptation occurs under the influence of the environment and selfdevelopment [15].

Analyzing the effects obtained by the risk management system as a result of adaptation, we distinguish the following cases: if $\Delta v\left(t_{0}, t_{k}\right)=0$, then there is no adaptation (the effect of the achieved goals is extremely low); at $\Delta v\left(t_{0}, t_{k}\right)>0$ adaptation has positive effects - a high level of goals achieved; when $\Delta v\left(t_{0}, t_{k}\right)<0$, adaptation gives a general decrease in efficiency - goals are not achieved [16].

In an adaptive risk management system there are no explosive hazards, i.e. such a moment is possible $t^{*}$, at which $\Delta v\left(t_{0}, t^{*}\right)<<v\left(t^{*}, t_{k}\right), t_{0}<t^{*}<t_{k}$, so catastrophic when $\Delta v\left(t_{0}, t^{*}\right)>\Delta v\left(t^{*}, t_{k}\right), t_{0}<t^{*}<t_{k}[17-18]$.

The forecast of the achievement of the risk management system of the stated objectives in the field of risk management is called the probability of an event in which at $\boldsymbol{t}_{\boldsymbol{k}}$ risk management system will produce effects of magnitude [19-20]. 


$$
v\left(t_{k}\right)=v_{k} \in V_{k} \subset V, \text { т.е. } p_{v}\left(t_{k}\right)
$$

\section{Conclusions}

The management process of a construction organization cannot be considered in a simplified manner, it determines the constancy of all management actions, the development and control of management decisions, the work of all managers, as well as the continuity of the implementation of the most important management functions: planning, organization and control. Thus, the construction of a two-stage dynamic model of risk management for a construction enterprise allows using a situational approach to management, as well as implementing a formal or instrumental principle inherent in any organizational system, taking into account the influence on its external and internal factors.

\section{References}

1. E. S. Wentzel, Probability theory: Textbook for students. Universities. - 9th ed., Sr, 576 (Moscow, Publishing center "Academy", 2003)

2. A. A. Voronov, Operations Research and Management, 128 (Moscow, Science, 1970)

3. D. I. Golenko, Statistical methods of network planning and management, 400 (Moscow, Science, 1968)

4. V. I. Denisov, Calculation of estimates of distribution parameters using tables of asymptotically optimal grouping, Application of computers in optimal planning and design, 3-17 (Novosibirsk: ed. NETI, 1981)

5. I. M. Artamonov, Yu. I. Deniskin, A. R. Deniskina, "Ensuring the unity and consistency of information as a tool for quality management", Quality and life, 4(16), 13-17 (2017)

6. V. Mager, V. Belousov, E. Desyatirikova, A. Polukazakov, S. Ivanov, I. Pocebneva, Proceedings of 2017 IEEE 2nd International Conference on Controls in Technical Systems, CTS 201714 November 2017, Article Number 8109503, 114-117, 2nd IEEE International Conference on Control Systems, CTS 2017; St. Petersburg; Russian Federation; 25 October 2017 to 27 October (2017) DOI: 10.1109 / CTSYS.2017.8109503

7. I. Pocebneva, Va. Belousov, Ia. Fateeva, Vb. Lukinov, Tc. Folomeeva, MATEC Web of Conferences 170, 13 June 2018, 011202017 International Science Conference on Sustainable Urban Development, SPbWOSCE 2017; Peter the Great St. Petersburg Polytechnic UniversitySt. Petersburg; Russian Federation; 20 December 2017 to 22 December (2017) DOI: 10.1051 / matecconf / 201817001120

8. Y. I. Bityukov, Y. I. Deniskin, G. Y. Deniskina, "Spline wavelets, for non-stationary linear control systems", Journal of Physics: Conference Series, 944(1), 30 January 2018, Article number 01201811th International Scientific and Technical Conference on Applied Mechanics and Dynamics Systems, AMSD 2017; Omsk; Russian Federation; 14 November 2017 to 16 November (2017) DOI: 10.1088 / 1742-6596/944/1/012018

9. V. E. Belousov, I. V. Komleva, I. V. Potsebneva, Models of tender risk management of the design organization, Bulletin of the Voronezh State Technical University, 7(8), 194-198 (2011)

10. V. E. Belousov, I. V. Pocebneva, V. V. Shabanov, Models of optimization of the structure of business processes for organizational management systems, Bulletin of the Voronezh State Technical University., 7(7), 189-193 (2011) 
11. V. E. Belousov, I. V. Pocebneva, N. M. Podvalnaya, A probable model of risk assessment of construction enterprises, Bulletin of the Voronezh State Technical University., 7(3), 189-192 (2011)

12. S. A. Chepelev, I. V. Pocebniva, V. Yu. Yershov, Development of a model system of forecasting risk using fuzzy logic, In the collection: Modern innovations in science and technology Collection of scientific papers of the 8th all-Russian scientific and technical conference with international participation. Responsible editor A. A. Gorokhov, 276280 (2018)

13. I. V. Fateev, I. V. Bakhtizina, Improving the efficiency and sustainability of construction projects through the application of the system macwell, In the collection: the Science of modern times: preserving the past - create a future Collection of scientific articles on the results of the International scientific and practical conference, 14-15 (2017)

14. Y. I. Deniskin, A. R. Deniskina, G. Yu. Deniskina, Numerical solution of systems of differential equations with variable coefficients for the tasks of quality management, Quality and life, 4(20), 137-140 (2018)

15. E. V. Ivanova, A. R. Deniskina, The application of information technology to improve the system of quality management of design organizations, Quality and life, 4-S(12), 279-281 (2016)

16. A. R. Deniskina, A. V. Romanyuk, Improving the process of supplier evaluation based on process approach, Quality and life, 4-S(12), 68-72 (2016)

17. V. E. Belousov, L. P. Mysovska, I. V. Pocebniva, A streaming algorithm for finding the early payment plan works for generalized network models, Quality and life, 2(18), 36-39 (2018)

18. I. P. Abrosimov, V. E. Belousov, I. V. Pocebniva, Heuristic resource allocation algorithm for cyclic network models, Quality and life, 3(19), 28-33 (2018)

19. V. I. Lepeshkin, I. V. Pocebniva, Modeling of technological processes of building production, In the book: aerospace technologies (ACT-2017) Theses of the XVIII International scientific and technical conference and school of young scientists, graduate students and students, 135-137 (2017)

20. I. V. Pocebniva, S. Alaa, A. S. Sobolev, A. S. Popov, Application of methods of computer simulation for managerial decision making, In the collection: young Science - the future of Russia Collection of scientific articles of the 2nd International scientific conference of promising developments of young scientists. In 5 volumes. Responsible editor A. A. Gorokhov, 144-148 (2017) 